\title{
ATITUDE DO CONSUMIDOR FRENTE À IRRADIAÇÃO DE ALIMENTOS ${ }^{1}$
}

\author{
Cléia Batista Dias ORNELLAS ${ }^{2, *}$, Maria Paula Junqueira GONÇALVES ${ }^{3}$, \\ Patrícia Rodrigues SILVA ${ }^{3}$, Renaldo Travassos MARTINS ${ }^{2}$
}

\begin{abstract}
RESUMO
Fatores econômicos e sociais como custo, disponibilidade e hábitos alimentares têm, tradicionalmente, influência sobre a escolha do consumidor. Atualmente, outros fatores como legislação, aumento da quantidade de refeições realizadas fora de casa e o emprego de novas tecnologias têm sido, também, parâmetros de decisão. Neste sentido, esclarecimentos a respeito da irradiação, proposta como uma alternativa na conservação de alimentos se faz necessária, uma vez que seu uso comercial tem sido lento em função de interpretações errôneas por parte dos consumidores. Sendo assim, o presente trabalho teve como objetivo realizar um levantamento do nível de conhecimento e aceitação da irradiação de alimentos, na cidade de Belo Horizonte (MG), bem como esclarecer ao consumidor o real conceito da irradiação de alimentos. Os resultados indicaram que 59,6\% dos entrevistados não sabiam que a irradiação é um método de conservação de alimentos e não souberam responder se consumiriam produtos irradiados, $16 \%$ acreditam que alimentos irradiados significam o mesmo que alimentos radioativos. Além disto, $89 \%$ dos entrevistados consumiriam alimentos irradiados se soubessem que a irradiação aumenta a segurança alimentar.
\end{abstract}

Palavras-chave: alimentos irradiados, atitude dos consumidores, conservação de alimentos.

\section{SUMMARY}

CUSTOMER ATTITUDE FRONT TO THE FOOD IRRADIATION. Economic and social factors as cost, availability and food habits usually influence the consumer's choice. Nowadays other factors like legislation, rising of meals eaten out-of-home and the application of new technologies have been affected the shopping decision. In this direction it's necessary to have more explanations about food irradiation as a method to conserve food. Its commercial use has been slow because most of the consumers misunderstands or has wrong belief about this technique. In such a manner, this work aimed at realizing a survey of knowledge and acceptance level of food irradiation in Belo Horizonte (MG), Brazil, and also to elucidate its real meaning to consumers. A total of 218 people were interviewed and the results showed that $59.6 \%$ of them haven't known that irradiation is a method to preserve food, thus they have no idea if they consume or not this kind of food. About $16 \%$ believe that irradiated food means the same of radioactive food. Besides that, $89 \%$ of people interviewed could become consumers of this product if they know that irradiation raises the food safety.

Keywords: irradiated foods, consumer's attitude, food preservation.

\section{1 - INTRODUÇÃO}

O mérito da irradiação está em sua capacidade de destruir microorganismos patogênicos e deteriorantes presentes nos alimentos. É empregada, ainda, para eliminar insetos e retardar o processo germinativo em produtos vegetais. Desta forma, há um aumento na segurança dos alimentos destinados ao consumo humano e uma redução nas perdas causadas por deterioração [6]. A irradiação é um processo físico que vem sendo estudado há vários anos, tendo o seu emprego regulamentado pelo Food and Drug Adminstration (FDA) desde 1963, para farinha de trigo e trigo destinados à alimentação humana, e suas aplicações têm sido guiadas sob as regras das Boas Práticas de Fabricação (BPF's) [5]. Posteriormente, nas décadas de 80 e 90, novas regulamentações surgiram com intuito de estender a utilização desta tecnologia para outros alimentos.

\footnotetext{
${ }^{1}$ Recebido para publicação em 9/6/2005. Aceito para publicação em 23/1/2006 (001548)

${ }^{2}$ Escola de Veterinária da Universidade Federal de Minas Gerais (UFMG)

Rua Jair Dutra de Moraes, 92/201

CEP 31260-290 - Belo Horizonte (MG)

E-mail: cleiaornellas@terra.com.br

${ }^{3}$ Centro Universitário de Belo Horizonte (UNI-BH), Curso de Engenharia de Alimentos

Avenida Professor Mário Werneck, 1.685

CEP 30455-610 - Belo Horizonte (MG)

*A quem a correspondência deve ser enviada
}

No Brasil, as primeiras pesquisas com irradiação de alimentos foram feitas da década de 50, pelo Centro de Energia Nuclear na Agricultura (Cena), em Piracicaba (SP). Mesmo com a permissão, em 1985, do uso da irradiação para conservação de alimentos, os estudos se restringiram quase que exclusivamente às instituições de pesquisas, uma vez que o País contava com um número restrito de especialistas [7].

A legislação brasileira segue as recomendações internacionais sugeridas pela Food and Agriculture Organization (FAO), Internatinal Atomic Energy Agengy (IAEA) e Codex Alimentarium, da ONU. Atualmente, todas as normas para o emprego desta tecnologia estão descritas na Resolução $\mathrm{n}^{\circ} 21$ [1], segundo a qual, qualquer alimento pode ser irradiado desde que sejam observados os limites mínimos e máximos da dosagem aplicada, sendo que a dose mínima deve ser suficiente para alcançar a finalidade pretendida e a máxima, inferior àquela que comprometeria as propriedades funcionais e/ou atributos sensoriais do alimento.

Apesar de toda aprovação e controle no emprego da irradiação, diversas barreiras ainda persistem e impedem que os alimentos irradiados alcancem a completa comercialização. Na verdade, não são barreiras de natureza técnica ou científica, mas relacionadas ao custo de sua utilização e de aceitação pelo consumidor.

Assim, apesar de cientificamente aceito como um excelente método de conservação de alimentos e de atualmente ser o único capaz de tornar inativos os patógenos em ali- 
mentos crus e congelados, o progresso no uso comercial da irradiação tem sido lento. Interpretações errôneas dos consumidores, que freqüentemente acham difícil avaliar os benefícios dessa técnica de processamento e a falta de informações têm limitado o uso desta tecnologia.

Em função disso, atitudes deverão ser implementadas começando pela conscientização dos consumidores em relação à segurança e benefícios obtidos por esta técnica e passando também por um estreitamento nas relações entre o governo e as indústrias do setor, que precisam ser fortalecidas. Para resultados mais eficazes, é preciso conhecer melhor o perfil dos segmentos envolvidos, por isso, sugere-se um levantamento desses dados utilizando-se recursos de pesquisas campo.

A pesquisa de opinião é uma ferramenta bastante eficaz para detectar, com precisão, posições e tendências dos diversos segmentos sociais. Baseada em dados científicos, esta atividade é um excelente instrumento para identificar problemas e buscar soluções [4].

Este tipo de pesquisa pode também ser utilizado para apontar, por exemplo, dados sobre o perfil e a imagem de marcas, instituições, entidades ou até mesmo de pessoas. Pode, ainda, avaliar a repercussão de novos acontecimentos, ações governamentais na opinião pública ou como reagiriam setores da população diante de novas tecnologias como o caso dos alimentos transgênicos e irradiados.

Este trabalho teve como objetivo a realização de pesquisa de opinião com consumidores para o levantamento do nível de conhecimento sobre a irradiação de alimentos e sua aceitação. Foi avaliada também a opinião das pessoas sobre a segurança dos mesmos e a credibilidade dos selos de certificação dos alimentos, além de educar o consumidor, a partir da distribuição de folheto informativo sobre o real benefício da irradiação como processo de conservação de alimentos.

\section{2 - MATERIAL E MÉTODOS}

O perfil do consumidor em relação aos alimentos irradiados foi traçado por meio de um questionário respondido por 218 entrevistados de diferentes bairros e classes sociais, na cidade de Belo Horizonte (MG). O método de coleta dos dados deu prioridade à entrevista pessoal, por ser um dos métodos mais empregados em pesquisa de opinião do consumidor. Para não haver a possibilidade de distorções tendenciosas a pesquisa foi realizada sem qualquer explicação prévia sobre o produto irradiado, sendo os esclarecimentos dados após a pesquisa. O folheto informativo distribuído aos entrevistados foi elaborado pelo Centro de Desenvolvimento de Tecnologia Nuclear (CDTN) da UFMG e cedido para realização desta pesquisa.

\section{3 - RESULTADOS E DISCUSSÃO}

Foram entrevistados 218 consumidores, sendo 65\% mulheres e $35 \%$ homens. A maioria dos entrevistados possui nível de instrução elevado, como evidencia a Figura 1, e renda superior a cinco salários mínimos (Figura 2). Estudos correlacionando estes e outros fatores, como a idade e o nível de conhecimento sobre a irradiação indicaram que este último não influenciou nos resultados obtidos, diferentemente do nível de educação e renda familiar, que apresentaram índice de correlação significativo. RESURRECCION et al [6] detectaram que a irradiação de alimentos é um problema maior para entrevistados de menor renda familiar e de menor nível de escolaridade. O mesmo resultado também foi verificado por CROWLEY, GABOURY AND WITT [3]. No entanto, estes pesquisadores ao correlacionar a idade dos entrevistados ao nível de conhecimento sobre a irradiação constataram correlação positiva entre eles.

Do total de entrevistados, 59,6\% não sabiam que a irradiação é um método de conservação de alimentos, e não souberam responder se consumiriam produtos irradiados.

Para $16 \%$, alimentos irradiados significam o mesmo que alimentos radioativos, evidenciando a falta de informação sobre a tecnologia de irradiação de alimentos. Em estudo similar, RESURRECCION et al. [6] constataram que $33 \%$ dos entrevistados, na cidade de Atlanta, nos Estados Unidos, e arredores tinham a idéia de que alimentos irradiados eram radioativos.

Outra evidência da falta de informação dos entrevistados é que $62 \%$ responderam não saber se a irradiação de alimentos pode trazer danos à saúde do consumidor e/ou ao meio ambiente. Em seu trabalho, RESURRECCION et al. [6] também verificaram falta de conhecimento sobre o assunto, uma vez que quase a metade dos entrevistados respondeu as perguntas com a opção "não sei” don't know, apesar das opções "verdadeiro" ou "falso" estarem presentes no questionário. Este fato deve ser explorado, no sentido de que os potenciais consumidores dos alimentos irradiados somente decidirão entre adquirir ou não efetivamente tais produtos se tiverem conhecimentos suficientes para a tomada de decisão.

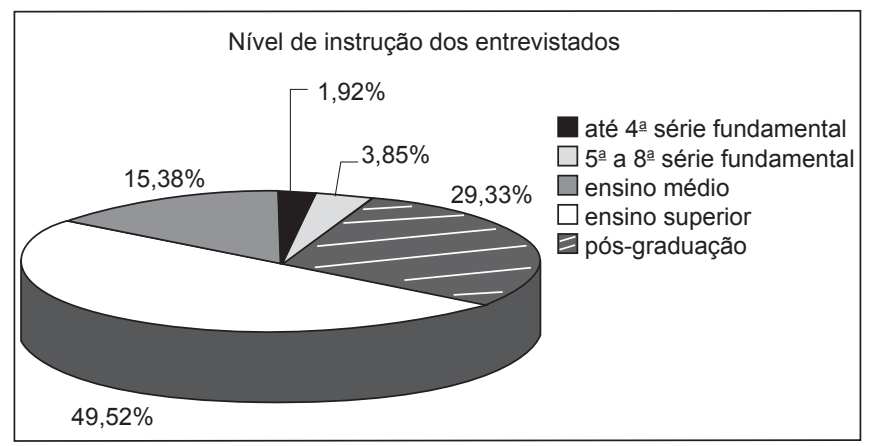

FIGURA 1 - Nível de instrução dos entrevistados

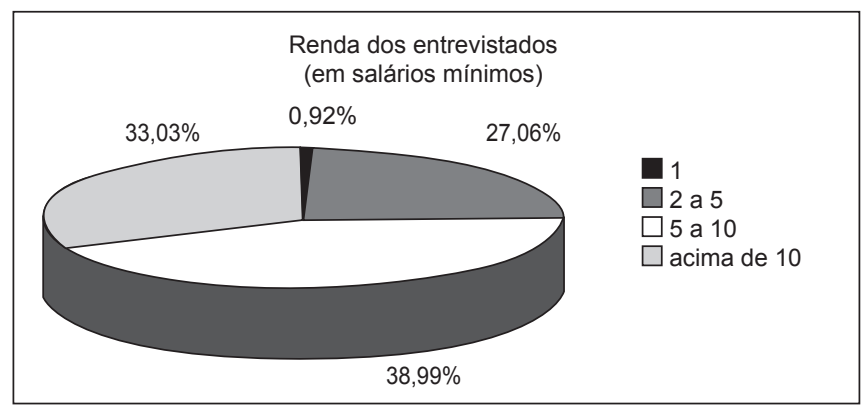

FIGURA 2 - Renda dos entrevistados 


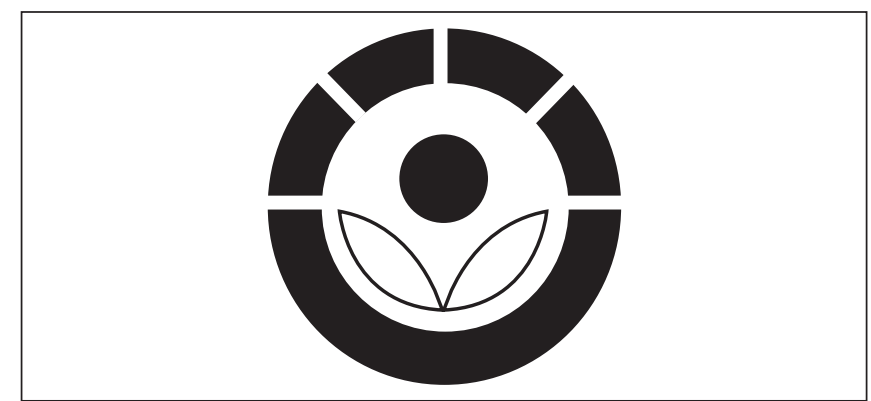

FIGURA 3 - Radura

A falta de conhecimento gera dúvidas e colabora para um posicionamento de repulsa diante dos alimentos tratados por esta nova tecnologia. CROWLEY, GABOURY AND WITT [3] constataram que há uma correlação positiva entre a consciência sobre o assunto e a disposição para comprar. No universo de 115 participantes de uma pesquisa de opinião, $85 \%$ já haviam ouvido falar sobre a irradiação de carnes e $70 \%$ deles demonstraram desejo em comprar este produto. No questionário com 19 participantes, houve justificativas positivas para a aquisição de carnes irradiadas, sendo a questão sanitária a mais importante delas.

Estudos de atitude e testes de compra demonstram que, quando é oferecida a oportunidade, os consumidores aceitam os alimentos irradiados. A maioria não tem tido esta opção e seus conhecimentos são limitados sobre esta tecnologia [2].

Do total de entrevistados, $45 \%$ afirmaram que observam os rótulos dos alimentos com freqüência, e a maioria afirmou que o atributo qualidade é o que determina a compra. Do total, $92 \%$ não conhecem o símbolo da irradiação, a radura, sendo que $16 \%$ comprariam alimentos irradiados pela influência do símbolo, mesmo sem saber seu significado, informando que a radura transmite confiança, segurança e qualidade, pela imagem da flor em coloração verde ( $F i$ gura 3). A etiqueta com o símbolo da irradiação bem com informações adicionais no rótulo foram indicadas como importante para $81 \%$ dos consumidores. Entretanto, o símbolo internacional e as declarações foram considerados pela metade dos entrevistados como insuficientes para informar sobre alimentos irradiados [6].

A pesquisa evidenciou que a falta de informação sobre a tecnologia da irradiação é um fator limitante do seu uso mais freqüente na indústria alimentícia brasileira, uma vez que $89 \%$ dos entrevistados consumiriam alimentos irradiados se soubessem que a irradiação aumenta a segurança alimentar contra inúmeras doenças (Figura 4). Este fato também foi constatado por CROWLEY, GABOURY AND WITT [3], que verificaram que quando há expectativa de benefícios para a saúde e os riscos são menores há maior disposição para compra.

\section{4 - CONCLUSÕES}

Os consumidores estão cada vez mais exigentes em relação à escolha de seus alimentos, e têm demonstrado grande interesse em conhecer novas tecnologias. Muitos

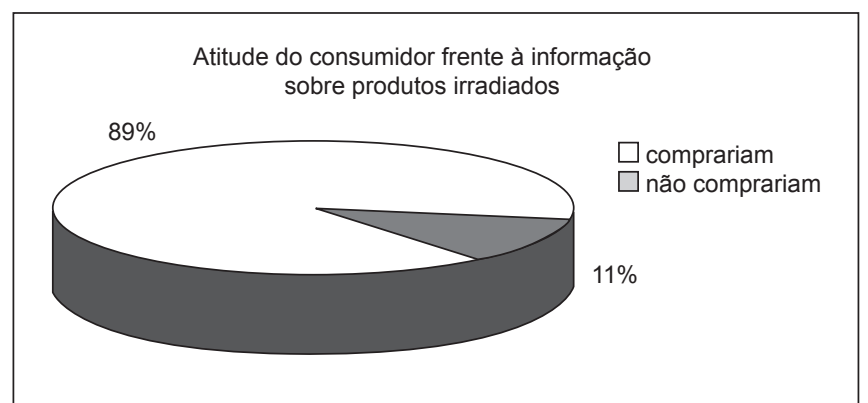

FIGURA 4 - Atitude do consumidor frente à informação sobre produtos irradiados

deles estão propensos a comprar alimentos obtidos ou tratados por métodos alternativos. Entretanto, a maioria gostaria de receber mais esclarecimentos sobre o assunto, evidenciando a necessidade de educação e divulgação mais ampla. Este proposta, se colocada em prática, poderá abrir o mercado para os alimentos tratados pela irradiação conforme verificado neste estudo, já que é a falta de informação a questão limitante da popularização desta tecnologia.

\section{5- REFERÊNCIAS BIBLIOGRÁFICAS}

[1] BRASIL. AGÊNCIA NACIONAL DE VIGILÂNCIA SANITÁRIA (ANVISA). Resolução $\mathrm{n}^{\circ} 21$, de 26 janeiro 2001. Disponível em: <http://anvisa.gov.Br/legis/resol/21_01rdc.htm > Acesso em: 24 mar. 2004.

[2] BRUHN, C.M. Strategies for communicating the facts on food irradiation to consumers. Journal of Food Protection, v. 58, n. 1, p. 213-216, 1995.

[3] CROWLEY, M.L., GABOURY, D. J., WITT, D. Chef's attitudes in North-Eastern US toward irradiation beef, Olestra, rBST and genetically engineered tomatoes. Food Service Technology, v. 2, p. 173-181, 2002.

[4] IBOPE. INSTITUTO BRASILEIRO DE OPINIÃO PÚBLICA E ESTATÍSTICA. Disponível em < http://www. ibope.com.br/calandraWeb/servlet/CalandraRedirect ?temp $=0 \&$ proj $=$ PortallBOPE \&pub $=\mathrm{T} \& \mathrm{db}=\mathrm{caldb}>$ Acesso em: $12 \mathrm{dez} .2004$.

[5] LAGUNAS- SOLAR, M.C. Radiation processing of foods: An overview of scientific principles and current status. Journal of Food Protection, v. 58, n. 2, p. 186-192, 1995.

[6] RESURRECCION, A.V.A, GALVEZ, F.C.F., FLETCHER, S.M., MISRA, S.K. Consumers attitudes towards irradiated food: results of a new study. Journal of Food Protection, v. 58, n. 2, p. 193-196, 1995.

[7] SANZ, E. Irradiação de alimentos pode aumentar exportaçóes de frutas brasileiras. Disponível em: <http://www.radiobras.gov.br/ct/1996/materia_270996_ 12.htm > Acesso em: 2 nov. 2004.

\section{6- AGRADECIMENTOS}

Centro de Desenvolvimento da Tecnologia Nuclear (CDTN/UFMG).

Centro Universitário de Belo Horizonte (UNI-BH).

Coordenação de Aperfeiçoamento de Pessoal de Nível Superior (Capes). 